\title{
Puerarin Inhibits FUNDC1-mediated Mitochondrial Autophagy and CSE-induced Apoptosis of Human Bronchial Epithelial Cells by Activating the PI3K/AKT/MTOR Signaling Pathway
}

\section{Li Wang}

Yan'an University Affiliated Hospital

Weizhou Jiang

Weifang Traditional Chinese Hospital

Jing Wang

Tai'an Maternal and Child Health Hospital

\section{Yuanyuan Xie}

Yan'an University Affiliated Hospital

Weisi Wang ( $\square$ hzwangweisi@163.com )

the Second Affiliated Hospital of Zhejiang Chinese Medical University https://orcid.org/0000-0002$3288-1960$

\section{Research}

Keywords: Chronic obstructive pulmonary disease, puerarin, mitochondrial autophagy, FUNDC1, $\mathrm{PI} 3 \mathrm{~K} / \mathrm{AKT} / \mathrm{mTOR}$ signaling pathway

Posted Date: June 5th, 2020

DOl: https://doi.org/10.21203/rs.3.rs-33044/v1

License: (9) This work is licensed under a Creative Commons Attribution 4.0 International License. Read Full License

Version of Record: A version of this preprint was published at Aging on February 8th, 2022. See the published version at https://doi.org/10.18632/aging.203317. 


\section{Abstract}

Background: Growing evidence suggests that the pathogenesis of chronic obstructive pulmonary disease (COPD) is related to mitochondrial autophagy mediated by FUN14 domain protein 1 (FUNDC1). Recent studies have shown that puerarin has protective effects against excessive oxidative damage in cells. We hypothesized that puerarin might be involved in the progression of COPD induced by cigarette smoke extract (CSE) by regulating FUNDC1-mediated mitochondrial autophagy.

Material/Methods: Different concentrations of puerarin were used to intervene in CSE-treated human bronchial epithelial cells (HBECs). MTT assay and flow cytometry were used to detect cell activity and apoptosis. The mitochondrial membrane potential (MMP) level, reactive oxygen species (ROS) content and ATP content were detected by flow cytometry or kits; Western Blotting was used to detect mitochondrial autophagy-related proteins expression such as DRP1, FUNDC1, PINK1, Parkin. Next, protein phosphatase inhibitor (PH0321) was used to inhibit the phosphorylation of FUNDC1, the changes of MMP, ROS, ATP and mitochondrial autophagy related proteins were detected. Furthermore, mitochondrial autophagy inhibitors (Mdivi) were used to block autophagy, and then the changes of mitochondrial autophagy, cell activity and apoptosis were detected, respectively. Finally, the changes of $\mathrm{PI} 3 \mathrm{~K} / \mathrm{AKT} / \mathrm{mTOR}$ signaling pathway-related proteins were detected by Western Blotting.

Results: Puerarin reversed cell activity decrease and apoptosis increase of CSE-induced HBECs, and down-regulated apoptosis-related proteins expression. Puerarin up-regulated MMP level and ATP content in CSE-induced HBECs, and down-regulated ROS content and mitochondrial autophagy-related proteins expression. PH0321 promotes mitochondrial autophagy by promoting the dephosphorylation of FUNDC1, puerarin inhibits the dephosphorylation of FUNDC1, up-regulated the MMP level and ATP content of CSEinduced HBECs, reduces ROS content and mitochondrial autophagy-related proteins expression. Compared with Mdivi group, the protein expression changes of PINK1 and Parkin in puerarin-treated group were not significantly different, and the cell activity decrease and apoptosis increase of CSEinduced HBECs were also significantly reversed by puerarin, which was not significantly different from Mdivi group. Puerarin significantly upregulated the protein expression of p-PI3K, p-AKT and p-mTOR, suggesting that puerarin may participate in the progression of COPD by activating the PI3K/AKT/mTOR signaling pathway.

Conclusions: Puerarin inhibits FUNDC1-mediated mitochondrial autophagy and CSE-induced apoptosis of HBECs by activating the PI3K/AKT/mTOR signaling pathway.

\section{Background}

Chronic obstructive pulmonary disease (COPD) is a common disease characterized by persistent airflow limitation, and its clinical manifestations are mainly chronic cough, shortness of breath, chest tightness or wheezing $[1,2]$. Persistent chronic inflammation induces the recurrence of tracheal wall injury and repair process, leading to airway remodeling, which is the main cause of irreversible progression of COPD 
$[3,4]$. The morbidity and mortality of COPD are increasing year by year, which seriously affects the working ability and quality of life of patients and causes a heavy socio-economic burden.

Smoking is the most important risk factor for COPD [5]. Cigarette smoke constantly stimulates the alveoli, leading to the elevation of endogenous reactive oxygen species (ROS) in inflammatory cells and epithelial cells, and mitochondrial damage, which in turn induces the imbalance between oxidative and antioxidant processes in cells [6]. It has been shown that mitochondrial autophagy is involved in cigarette smoke extract (CSE) induced regulation of human bronchial epithelial cells (HBECs), thus affecting the pathogenesis of COPD $[7,8]$. In COPD progression, mammalian mitotic receptor FUN14 domain protein 1 (FUNDC1) is closely related to autophagy and apoptosis of hypoxic cells, which can participate in mitosis through interaction with mitochondria [9]. Wen et al found that silencing the FUNDC1 gene in CSEinduced HBECs could inhibit mitochondrial autophagy and cell apoptosis by interacting with DRP1, and slow down the COPD process [10].

At present, most of the acute treatment options for COPD are antibiotics, bronchodilators, glucocorticoids and expectorants, but these treatment measures could not effectively delay or prevent the recurrence and progressive progress of the disease. Studies have shown that flavonoids have anti-inflammatory and antioxidant properties, which can effectively improve lung function and reduce inflammatory cell infiltration in chronic airway inflammation [11-13]. Gao et al showed that Icariside II could protect PC12 cells from $\mathrm{H}_{2} \mathrm{O}_{2}$-induced death by inhibiting mitochondrial-mediated autophagy [14].

Puerarin is an isoflavone compound isolated from the dried root of pueraria lobata, a traditional Chinese medicine. Its medicinal value is benefited from its wide range of pharmacological properties, such as vasodilation, cardio-protection, antioxidant, anti-apoptotic and reducing insulin resistance [15]. Puerarin plays a protective role against cell damage caused by pathological factors [16]. Puerarin has been reported to reduce Caspase-3 expression by activating PI3K/AKT signaling pathway, and significantly improve tumor necrosis factor- $a$ (TNF-a) induced apoptosis in PC12 cells [17]. In addition, Chen et al showed that puerarin can directly act on myocytes, alleviate mitochondrial dysfunction, mitosis and inflammatory response induced by palmitate, thereby contributing to the improvement of insulin sensitivity and the improvement of impaired insulin signaling in skeletal muscle and isolated myotubes of diabetic animals [18]. As mentioned above, puerarin may play an active role in targeted therapy of chronic obstructive pneumonia disease.

\section{Materials And Methods}

\section{Cell culture}

The HBECs were purchased from the American Type Culture Collection (ATCC; https://www. atcc.org/) and stored in Dulbecco's modified Eagle's medium (DMEM, Sigma-Aldrich Chemical Company, St Louis, MO) containing 10\% FBS (Sigma-Aldrich Chemical Company) and penicillin-streptomycin solution (100x; Thermo Fisher Scientific Inc, Waltham, MA). Puerarin was provided by Shanghai McLean Biochemical 
Technology Company, batch number: $\mathrm{C} 11030370$, purity $>98 \%$. The suspension was prepared with sterilized saline at the concentration of $50 \mu \mathrm{g} / \mathrm{mL}, 100 \mu \mathrm{g} / \mathrm{mL}$ and $200 \mu \mathrm{g} / \mathrm{mL}$ and stored in a refrigerator at $4^{\circ} \mathrm{C}$ in the dark for subsequent experiments.

\section{Preparation of 100\% Cigarette Smoke Extract}

The cigarettes used in this experiment were all Nanjing cigarettes of a brand. The cigarette collection system was assembled according to the cigarette support tube, gas collection bottle, buffer bottle and peristaltic pump. During gas collection, after adding about $10 \mathrm{~mL}$ DMEM culture medium into the gas collection bottle, start the peristaltic pump, adjust the speed about $160 \mathrm{r} / \mathrm{min}$, at this time the gas flow rate is about $600 \mathrm{~mL} / \mathrm{min}$, ignite 1.5 cigarettes in turn, and shake the gas collection bottle continuously to dissolve the cigarette smoke as soon as possible. After the cigarette is burned out, the liquid obtained is recorded as $100 \%$ CSE, placed on the ultra-clean table for filtration and sterilization. After that, the CSE solution was added into DMEM to prepare the CSE mixture, which held a concentration of $20 \%$ and used as soon as possible within 30 minutes.

\section{Rna Extraction And Rt-qpcr}

Total RNA was isolated from HEBCs by using the TRIzol (Invitrogen, Carlsbad, CA, USA)). Single-stranded cDNA was synthesized with the PrimeScript Reagent Kit (Promega, USA). Real-time PCR was conducted by using SYBR Premix Ex TaqTM Kit (Applied Biosystems, Foster City, CA, USA). The reaction was run in ABI7500 Real-time PCR system (Applied Biosystems, Carlsbad, CA). GAPDH was used as an endogenous control. Briefly, $2 \mu \mathrm{L}$ of cDNA was added to $10 \mu \mathrm{L}$ of the $1 \times$ SYBR green PCR master mix with $0.4 \mu \mathrm{L}$ of Taq polymerase enzyme (RiboBio, China), $0.8 \mu \mathrm{L}$ of each primer and $6 \mu \mathrm{L} \mathrm{ddH}_{2} \mathrm{O}$ to a final volume of $20 \mu \mathrm{L}$. The RT-PCR cycling conditions consisted of: $95^{\circ} \mathrm{C}$ for $10 \mathrm{~min}$; then 35 cycle amplification for $20 \mathrm{~s}$ at $95^{\circ} \mathrm{C}, 30 \mathrm{~s}$ at $55^{\circ} \mathrm{C}, 15 \mathrm{~s}$ at $72{ }^{\circ} \mathrm{C}$; followed by $1 \mathrm{~min}$ at $72{ }^{\circ} \mathrm{C}$. The primers used in this study were synthesized from Sangon Biotech (Shanghai, China). The level of mRNA was normalized to $\beta$-actin expression using the $2^{-\Delta \Delta C t}$ method.

\section{Western Blot Analysis}

The cells were lysed for $20 \mathrm{~min}$ on ice in ice-cold lysis buffer (Roche). The lysates were centrifuged at $12,000 \times \mathrm{g}$ for $20 \mathrm{~min}$ at $4^{\circ} \mathrm{C}$ to obtain a clear lysate. The protein content of each sample was determined using the BCA Protein Assay Kit (Thermo Scientific). Then, equal amounts of proteins ( $15 \mu \mathrm{g} /$ lane) were separated on a $12 \%$ sodium dodecyl sulfate polyacrylamide gel electrophoresis (SDS-PAGE) and transferred to polyvinylidenedifluoride (PVDF) membranes (Bio-Rad, Hercules, CA, USA). The membranes were blocked in $5 \%(\mathrm{w} / \mathrm{v})$ nonfat dry milk in TBST (Tris-buffered saline- $0.1 \%$ Tween) at $25^{\circ} \mathrm{C}$ for $3 \mathrm{~h}$ and then incubated with the following primary antibodies: rabbit monoclonal anti- $\beta$-actin antibody (1:900, Abcam, ab179467), rabbit monoclonal anti-PINK1 antibody (1:1000, Abcam, ab216144), mouse 
monoclonal anti-Parkin antibody (1:1500, Abcam, ab77924), rabbit polyclonal anti-Cleaved Casepase3 antibody (1:1100, Abcam, ab2302), rabbit monoclonal anti-Bax antibody (1:2000, Abcam, ab32503), rabbit monoclonal anti-DRP1 antibody (1:1000, Abcam, ab184247), rabbit polyclonal anti-FUNDC1 antibody (1:500, Abcam, ab224722), rabbit monoclonal anti-PI3K antibody (1:1000, Abcam, ab32089), rabbit polyclonal anti-AKT antibody (1:1500, Abcam, ab179463), rabbit polyclonal anti-mTOR antibody (1:2000, Abcam, ab2732). The bands were visualized using horseradish peroxidase (HRP) conjugated goat anti-rabbit lgG (1:15000, Boster) prior to the ECL protocol (Amersham Biosciences, Piscataway, NJ, USA).

\section{Detection Of Mmp Level By Flow Cytometry}

Cells were collected by $0.25 \%$ trypsin (containing EDTA) digestion method; 200 mesh screen filtration, cell counting, $1.5 \times 10^{5}$ cells were collected; cells were resuspended by adding I mL JC- 1 working solution (Ix) under dark conditions, incubated at $37{ }^{\circ} \mathrm{C}$ for $20 \mathrm{~min}$ in dark, shaken once at $2 \varangle 3 \mathrm{~min}$ intervals; and centrifuged at $4{ }^{\circ} \mathrm{C}$ for $1500 \mathrm{rmp}$; the hearts were washed twice with JC-1 staining buffer (Ix) for $5 \mathrm{~min}$, and the cells were resuspended with $300 \mu \mathrm{L} J \mathrm{C}-1$ staining buffer $(\mathrm{I} \times)$ for flow cytometry detection.

\section{Detection Of Ros Content By Flow Cytometry}

Cells were collected by $0.25 \%$ trypsin (containing EDTA) digestion method; cells were filtered through 200 mesh screen, counted, and $1.5 \times 10^{5}$ cells were collected; preparation of DCFH-DA working solution: incomplete cell culture solution is 1:1000; cells were resuspended by adding DCFH-DA working solution under dark conditions, and incubated at 37 degrees in the dark for $20 \mathrm{~min}$; cells were washed three times with incomplete cell culture medium and resuspended with $300 \mu \mathrm{L}$ of incomplete cell culture medium for flow cytometry detection.

\section{Atp Content Detection}

Cells were collected by digestion with $0.25 \%$ trypsin (including EDTA); ATP lysate was added, mixed with shaking, ice bath for $10 \mathrm{~min}, 4^{\circ} \mathrm{C}$ freezing centrifuge for $12000 \times \mathrm{g}$ Centrifuge for $5 \mathrm{~min}$, extract the supernatant; prepare ATP detection working solution with ATP detection reagent: ATP detection diluent $=$ 1:4 in dark condition; $100 \mu \mathrm{L}$ working fluid was added into 96-well plate, stand at room temperature for $2 \bowtie 5$ minutes, then add standard diluent and sample $20 \mu \mathrm{L}$, and quickly mixed with a pipette gun; RUL was measured on a microplate reader and ATP concentration was calculated; protein concentration was measured at the same time, and finally ATP concentration: protein concentration was used as the final result, unit was $\mathrm{nmoL} / \mathrm{mg}$.

\section{Statistical analysis}


All statistical analyses were performed using the SPSS software (ver. 13.0; SPSS, Chicago, IL). The quantitative data derived from three independent experiments are expressed as mean \pm SEM. Significance was determined by one-way ANOVA or t-test. Values of $P<0.05$ were considered statistically significant.

\section{Results}

\section{Puerarin promotes the proliferation and inhibits apoptosis of CSE-induced HBECs}

To further explore the effect of puerarin on the proliferation and apoptosis of CSE-induced HBECs, different concentrations of puerarin were used to intervene in model cells. MTT assay results showed that the gradual increase of puerarin concentration could significantly promote the proliferation of HBECs compared with $20 \%$ CSE treatment alone (Fig. 1A). Next, the results of flow cytometry showed that the apoptosis of HBECs treated with $20 \%$ CSE was significantly increased, but this trend was reversed after puerarin intervention at different concentrations, and the inhibition of apoptosis by high concentrations of puerarin was more significant (Fig. 1B). Western blotting results suggested that the expression of apoptosis-related genes, including Cleaved caspase 3 and Bax, decreased after puerarin intervention and was significantly correlated with the concentration (Fig. 1C).

\section{Puerarin Inhibits Mitochondrial Autophagy Of Cse-induced Hbecs}

To clarify the effect of puerarin on mitochondria, puerarin with concentrations of $50 \mu \mathrm{g} / \mathrm{ml}, 100 \mu \mathrm{g} / \mathrm{ml}$ and $200 \mu \mathrm{g} / \mathrm{ml}$ was used to interfere with $20 \%$ CSE-induced HBECs, respectively. The changes of MMP level were detected by flow cytometry after labeling with JC-1 probe and the results showed that MMP level was significantly decreased after $20 \%$ CSE treatment, suggesting early apoptosis of cells. However, the decrease of membrane potential was gradually reversed after puerarin intervention at different concentrations, and was significantly correlated with puerarin concentration (Fig. 2A). In addition, the results of flow cytometry suggested that ROS levels in HBECs increased significantly after $20 \%$ CSE treatment, while puerarin intervention could down-regulate ROS levels in cells, which was significantly correlated with the concentration (Fig. 2B). Mitochondria are the main site of ATP synthesis. Treating cells with $20 \%$ CSE leads to damage of mitochondrial structure and function, and ATP content in cells decreases. Puerarin at different concentrations can significantly improve mitochondrial damage and increase the amount of ATP synthesis (Fig. 2C). Western blotting was used to detect the expression levels of mitochondrial autophagy-related proteins. The results showed that the protein expression of PINK1 and Parkin decreased significantly with the increase of puerarin intervention concentration compared with $20 \%$ CSE treatment alone, suggesting that puerarin had an inhibitory effect on mitochondrial autophagy (Fig. 2D). 


\section{Puerarin Down-regulates The Expression Of Drp1 And Fundc1}

To investigate whether CSE-induced autophagy is associated with DRP1 and FUNDC1, the expression of DRP1 and FUNDC1 was measured. The results of RT-PCR and Western blotting showed that DRP1 mRNA and protein expression were significantly increased after treatment of cells with $20 \%$ CSE, but this trend was reversed with the increasing concentration of puerarin intervention (Fig. 3A and 3B). Similarly, the expression of FUNDC1 mRNA and protein was significantly up-regulated in $20 \%$ CSE-treated group, but its expression was down-regulated after puerarin intervention and significantly correlated with the concentration (Fig. 3C and 3D). In addition, Western blotting results showed that p-FUNDC1 protein expression was significantly down-regulated after $20 \%$ CSE treatment, and puerarin intervention at different concentrations could significantly reverse its expression, which was correlated with the concentration. This suggests that FUNDC1 inhibits mitochondrial autophagy in the form of phosphorylation (Fig. 3D).

\section{Puerarin Prevents Mitochondrial Autophagy By Inhibiting Dephosphorylation Of Fundc1}

To further explore whether puerarin prevents mitochondrial autophagy by inhibiting the dephosphorylation of FUNDC1, PH0321, a protein phosphatase inhibitor, was used to inhibit the dephosphorylation of FUNDC1. Western blotting results showed that the expression of p-FUNDC1 was decreased and mitochondrial autophagy was promoted after PH0321 treatment. The expression of pFUNDC1 was significantly increased after intervention with high concentration of puerarin, which inhibited mitochondrial autophagy (Fig. 4A). Next, the results of flow cytometry suggested that PH0321 treatment alone significantly decreased MMP level, and MMP level significantly increased after highconcentration puerarin intervention (Fig. 4B). In addition, the content of ROS in cells was significantly increased after $\mathrm{PH} 0321$ treatment, but this trend was reversed by high concentration puerarin intervention, which significantly decreased the content of ROS in cells (Fig. 4C). Furthermore, the inhibitory effect of PH0321 on ATP synthesis was reversed after high-concentration puerarin intervention (Fig. 4D). Western blotting results showed that the expression of mitochondrial autophagy-related proteins such as PINK1 and Parkin was significantly upregulated after PH0321 treatment, and the expression of these two proteins was significantly downregulated after high-concentration puerarin intervention (Fig. 4E). The above results indicate that puerarin prevents mitochondrial autophagy by inhibiting the dephosphorylation of FUNDC1.

\section{Puerarin inhibits apoptosis of CSE-induced HBECs by inhibiting mitochondrial autophagy}

To further explore the effect of puerarin on apoptosis after inhibiting mitochondrial autophagy, we blocked the process of autophagy by using Mdivi, a mitochondrial division protein, as an inhibitor of mitochondrial autophagy. The results of Western blotting suggested that the expression of related 
proteins, such as PINK1 and Parkin, was significantly decreased after the mitochondrial autophagy process was blocked, and the changes of both proteins were consistent with the high concentration puerarin intervention (Fig. 5A). Furthermore, MTT assay was used to detect cell viability and the results showed that the inhibition of $20 \%$ CSE on cell viability was reversed after treatment with mitochondrial autophagy inhibitors, which showed that the cell viability was the same as that that in the high concentration puerarin intervention group (Fig. 5B). Next, autophagy inhibitor treatment significantly reduced the apoptosis rate, and the expression of apoptosis- related proteins such as Cleaved caspase3 and Bax was also significantly down-regulated, which showed the same trend as the high-concentration puerarin intervention (Fig. 5C and 5D).

\section{Puerarin inhibits mitochondrial autophagy and apoptosis in CSE-induced HBECs by activating the $\mathrm{PI} 3 \mathrm{~K} / \mathrm{AKT} / \mathrm{mTOR}$ signaling pathway}

The PI3K/AKT/mTOR signaling pathway widely exists in a variety of cells and participates in a variety of physiological and pathological processes in vivo, and its activation has an inhibitory effect on cell autophagy. To further explore whether inhibits mitochondrial autophagy and apoptosis in CSE-induced HBECs by activating the PI3K/AKT/mTOR signaling pathway, 3-MA, a PI3K inhibitor, was used to block the pathway. It was found that the protein expression of p-PI3K, p-AKT and p-mTOR were significantly reduced in CSE-induced HBECs, while its expression was significantly reversed after high concentration puerarin intervention, suggesting that puerarin can activate the PI3K/AKT/mTOR signaling pathway (Fig. 6A). Next, we blocked PI3K with 3-MA, and as expected, the expression of p-PI3K, p-AKT, and p-mTOR in cells was significantly decreased after pathway blockade compared with the high-concentration puerarin-treated group, and this signaling pathway was inhibited (Fig. 6B). Western blotting results showed that the expression of mitochondrial autophagy-related proteins such as PINK1 and Parkin increased significantly after the pathway was blocked (Fig. 6C), and the expression of apoptosis-related proteins such as Cleaved Casepase3 and Bax was also significantly upregulated (Fig. 6D). The above results further verified that the inhibition of puerarin on mitochondrial autophagy and apoptosis in CSEinduced HBECs was accomplished by activating the PI3K/AKT/mTOR signaling pathway.

\section{Discussion}

Mitochondria are energy factories in eukaryotic cells, which can provide energy for various physiological activities of the body [7]. With the occurrence of mitochondrial oxidative phosphorylation, a large number of ROS will be produced, and excessive ROS will lead to mitochondrial damage, causing apoptosis [19]. Therefore, clearance of damaged mitochondria through autophagy, a degradation pathway, is essential to maintain the stability of the intracellular environment.

FUNDC1 plays a key regulatory role in the biological process of mitochondrial autophagy. FUNDC1 is localized on the outer mitochondrial membrane and participates in mitochondrial autophagy caused by hypoxia and reduced mitochondrial membrane potential, in which the phosphorylated form of FUNDC1 inhibits autophagy while dephosphorylation promotes autophagy [20,21]. Wang et al found that 
mitochondrial DNA copy number and mitochondrial protein expression were significantly decreased in Ginkgolic acids-treated mouse bone marrow stromal cells, but FUNDC1 gene knockout restored Ginkgolic acids-induced changes in mitochondrial mass loss and mitochondrial membrane potential loss[22]. Another study showed that FUNDC1 plays an important regulatory role in hypoxia-induced PC12 neuroautophagy and apoptosis, and overexpression of FUNDC1 can promote autophagy and then prevent neuronal apoptosis [20]. In myocardial ischemia-reperfusion injury model, FUNDC1-mediated mitochondrial autophagy was inhibited, damaged mitochondria could not be cleared in time and accumulated in a large amount in cells, leading to microvascular endothelial cell apoptosis and barrier dysfunction [23]. In addition, some studies have found that hydrogen peroxide can up-regulate the expression of FUNDC1 by activating ERK1/2 signaling, triggering mitochondrial autophagy, making laryngeal cancer cells more suitable for survival [24].

In recent years, more and more studies have found that mitochondrial autophagy plays an important regulatory role in the pathogenesis of COPD. Among them, Araya Jun et al found that CS-exposed mouse airway epithelial cells showed impaired mitochondrial volume accumulation, increased oxidative modification and accelerated cell senescence, and experimental animals showed enhanced airway wall thickening and emphysema changes [25]. The upstream regulatory mechanism of mitochondrial autophagy is still unclear, and it is generally accepted that mitochondrial division is one of the important upstream regulatory factors of mitochondrial autophagy. Among them, DRP1 is a key executor. Normally, mitochondria maintain cell energy supply by controlling their number through division, but excessive mitochondrial division leads to the production of a large number of dysfunctional mitochondrial fragments, which in turn triggers mitochondrial autophagy [26-28]. In this study, we found that CSEinduced HEBCs could significantly upregulate the expression of DRP1 and FUNDC1, as well as the expression of autophagy-related proteins and apoptosis-related proteins, indicating that mitochondrial autophagy plays a positive regulatory role on apoptosis.

At present, there are few studies on the regulatory relationship between puerarin and DRP1 or FUNDC1, but the effect of flavonoids on mitochondrial autophagy through regulating DRP1 has been widely reported. Isoliglycyrrhizin isolated from Licorice attenuates neuronal cell death mediated by loss of mitochondrial membrane potential by promoting dephosphorylation of DRP1, inhibiting ROS generation and calcium ion levels in cells, and preventing glutamate-induced mitochondrial fission [29]. In a mouse model of Alzheimer's disease, icariin treatment led to a decrease in DRP1, promoted mitochondrial transport, and protected mitochondria from fragmentation, suggesting that it may be a potential therapeutic supplement for neurodegenerative diseases associated with Alzheimer's disease and other mitochondrial dysfunctions [30]. Other studies have reported that kaempferol protects neurons from succinate-mediated ischemic injury by inhibiting mitochondrial division by activating the AKT axis [31].

We found that different concentrations of puerarin could significantly reverse the damage of CES on HEBCs, which was manifested by restoring MMP level, reducing ROS generation, promoting ATP synthesis, reducing mitochondrial autophagy and inhibiting apoptosis. We further found that puerarin inhibited mitochondrial autophagy and mitigated cell injury by inhibiting the expression of DRP1 and 
FUNDC1, and this protective effect may be closely related to the activation of PI3K/AKT/mTOR signaling pathway. Puerarin has been reported to alleviate oxidative stress by inhibiting lead-induced renal cell apoptosis, restoring the balance between $\mathrm{Bax}$ and $\mathrm{Bcl}-2$, and reducing mitochondrial Cyt $\mathrm{C}$ release through activating the PI3K/AKT/eNOS pathway[32]. Puerarin can attenuate mitochondrial ultrastructure destruction, change MMP level to reverse cadmium-induced ATP depletion, and increase Bcl-2/Bax ratio, blocking Casepase3 activation [33]. A recent study claimed that the tuber extract of Alisma orientalis alleviated airway inflammation and emphysema phenotype in mice by activating mTOR and inhibiting autophagy [34].

\section{Conclusion}

In conclusion, our study shows that puerarin can inhibit FUNDC1-mediated mitochondrial autophagy and bronchial epithelial cell apoptosis by activating the PI3K/AKT/mTOR signaling pathway to achieve cytoprotective effects, which provides a new idea for the treatment of COPD.

\section{Declarations}

\section{Conflicts of Interest}

The authors declare that there are no conflicts of interest.

\section{Authors' contributions}

Li Wang and Weizhou Jiang finished acquisition and analysis of the data, as well as wrote the manuscript; Jing Wang and Weisi Wang participated and performed data analysis in this work; Yuanyuan Xie conceived this project and revised the manuscript.

\section{Funding}

None

\section{Availability of data and materials}

Not applicable

\section{Acknowledgements}

None

\section{Ethics approval and consent to participate}

None

\section{Consent for publication}


Not applicable.

\section{References}

1. Zhai PY, Li HX, Zhao RF, Li XQ, Wang HQ. [Clinical characteristics of pneumoconiosis complicated with chronic obstructive pulmonary disease]. Zhonghua Lao Dong Wei Sheng Zhi Ye Bing Za Zhi. 2019;37:899-902.

2. Ren $Y$, Zhang $Y$, Fan $L$, Jiao Q, Wang $Y$, Wang $Q$. The cullin4A is up-regulated in chronic obstructive pulmonary disease patient and contributes to epithelial-mesenchymal transition in small airway epithelium. Respir Res. 2019;20:84.

3. Tang K, Zhao J, Xie J, Wang J. Decreased miR-29b expression is associated with airway inflammation in chronic obstructive pulmonary disease. Am J Physiol Lung Cell Mol Physiol. 2019;316:L621-1629.

4. Sugiyama Y, Asai K, Yamada K, et al. Decreased levels of irisin, a skeletal muscle cell-derived myokine, are related to emphysema associated with chronic obstructive pulmonary disease. Int $\mathrm{J}$ Chron Obstruct Pulmon Dis. 2017;12:765-72.

5. Chen X, Li Y, Hua C, et al. Establishment of rapid risk assessment model for cigarette smoke extract exposure in chronic obstructive pulmonary disease. Toxicol lett. 2019;316:10-9.

6. Pouwels SD, Hesse L, Faiz A, et al. Susceptibility for cigarette smoke-induced DAMP release and DAMP-induced inflammation in COPD. Am J Physiol Lung Cell Mol Physiol. 2016;311:L881-I892.

7. Jiang $Y$, Wang $X$, Hu D. Mitochondrial alterations during oxidative stress in chronic obstructive pulmonary disease. Int J Chron Obstruct Pulmon Dis. 2017;12:1153-62.

8. Ryter SW, Rosas IO, Owen CA, et al. Mitochondrial Dysfunction as a Pathogenic Mediator of Chronic Obstructive Pulmonary Disease and Idiopathic Pulmonary Fibrosis. Ann Am Thorac Soc. 2018;15:266-s272.

9. Yao RQ, Ren C, Xia ZF, Yao YM. Organelle-specific autophagy in inflammatory diseases: a potential therapeutic target underlying the quality control of multiple organelles. Autophagy. 2020:1-17.

10. Wen W, Yu G, Liu W, et al. Silencing FUNDC1 alleviates chronic obstructive pulmonary disease by inhibiting mitochondrial autophagy and bronchial epithelium cell apoptosis under hypoxic environment. J Cell Biochem. 2019;120(10):17602-15.

11. Lu MC, Yang MD, Li PC, et al. Effect of Oligomeric Proanthocyanidin on the Antioxidant Status and Lung Function of Patients with Chronic Obstructive Pulmonary Disease. In vivo (Athens, Greece). $2018 ; 32: 753-758$.

12. Mitani A, Azam A, Vuppusetty C, Ito K, Mercado N, Barnes PJ. Quercetin restores corticosteroid sensitivity in cells from patients with chronic obstructive pulmonary disease. Exp Lung Res. 2017;43:417-25.

13. Liu XJ, Bao HR, Zeng XL, Wei JM. Effects of resveratrol and genistein on nuclear factorkappaB, tumor necrosis factoralpha and matrix metalloproteinase 9 in patients with chronic obstructive 
pulmonary disease. Mol Med Rep. 2016;13:4266-72.

14. Gao J, Deng Y, Yin C, et al. Icariside II, a novel phosphodiesterase 5 inhibitor, protects against $\mathrm{H} 2 \mathrm{O} 2$ induced PC12 cells death by inhibiting mitochondria-mediated autophagy. J Cell Mol Med. 2017;21:375-86.

15. Bacanli M, Basaran AA, Basaran N. The antioxidant, cytotoxic, and antigenotoxic effects of galangin, puerarin, and ursolic acid in mammalian cells. Drug Chem Toxicol. 2017;40:256-62.

16. Xie N, Wang C, Lian Y, Wu C, Zhang H, Zhang Q. Puerarin protects hippocampal neurons against cell death in pilocarpine-induced seizures through antioxidant and anti-apoptotic mechanisms. Cell Mol Neurobiol. 2014;34:1175-82.

17. Liang F, Xie S. Puerarin prevents tumor necrosis factor-alpha-induced apoptosis of PC12 cells via activation of the PI3K/Akt signaling pathway. Exp Ther Med. 2017;14:813-8.

18. Chen X, Yi L, Song S, et al. Puerarin attenuates palmitate-induced mitochondrial dysfunction, impaired mitophagy and inflammation in L6 myotubes. Life Sci. 2018;206:84-92.

19. Li X, Yang H, Sun $H$, et al. Taurine ameliorates particulate matter-induced emphysema by switching on mitochondrial NADH dehydrogenase genes. Proceed Natl Acad Sci U S A. 2017;114:E9655-64.

20. Wang L, Wang P, Dong H, et al. Ulk1/FUNDC1 Prevents Nerve Cells from Hypoxia-Induced Apoptosis by Promoting Cell Autophagy. Neurochem Res. 2018;43:1539-48.

21. Wu W, Li W, Chen H, Jiang L, Zhu R, Feng D. FUNDC1 is a novel mitochondrial-associated-membrane (MAM) protein required for hypoxia-induced mitochondrial fission and mitophagy. Autophagy. 2016;12:1675-6.

22. Wang W, Wang M, Ruan Y, et al. Ginkgolic Acids Impair Mitochondrial Function by Decreasing Mitochondrial Biogenesis and Promoting FUNDC1-Dependent Mitophagy. J Agric Food Chem. 2019;67:10097-106.

23. Zhou $\mathrm{H}$, Wang J, Zhu P, et al. NR4A1 aggravates the cardiac microvascular ischemia reperfusion injury through suppressing FUNDC1-mediated mitophagy and promoting Mff-required mitochondrial fission by CK2alpha. Basic Res Cardiol. 2018;113:23.

24. Hui L, Wu H, Wang TW, Yang N, Guo X, Jang XJ. Hydrogen peroxide-induced mitophagy contributes to laryngeal cancer cells survival via the upregulation of FUNDC1. Clin Translat Oncol. 2019;21:596606.

25. Araya J, Tsubouchi K, Sato N, et al. PRKN-regulated mitophagy and cellular senescence during COPD pathogenesis. Autophagy. 2019;15:510-26.

26. Wang J, Zhu P, Li R, Ren J, Zhou H. Fundc1-dependent mitophagy is obligatory to ischemic preconditioning-conferred renoprotection in ischemic AKI via suppression of Drp1-mediated mitochondrial fission. Redox Biol. 2020;30:101415.

27. Wu W, Lin C, Wu K, et al. FUNDC1 regulates mitochondrial dynamics at the ER-mitochondrial contact site under hypoxic conditions. EMBO J. 2016;35:1368-84. 
28. Chen M, Chen Z, Wang Y, et al. Mitophagy receptor FUNDC1 regulates mitochondrial dynamics and mitophagy. Autophagy. 2016;12:689-702.

29. Lee DG, Min JS, Lee HS, Lee DS. Isoliquiritigenin attenuates glutamate-induced mitochondrial fission via calcineurin-mediated Drp1 dephosphorylation in HT22 hippocampal neuron cells. Neurotoxicology. 2018;68:133-41.

30. Chen Y, Han S, Huang X, Ni J, He X. The Protective Effect of Icariin on Mitochondrial Transport and Distribution in Primary Hippocampal Neurons from 3x Tg-AD Mice. Int J Mol Sci 2016; 17(2).

31. Wu B, Luo H, Zhou X, et al. Succinate-induced neuronal mitochondrial fission and hexokinase II malfunction in ischemic stroke: Therapeutical effects of kaempferol. Biochim Biophys Acta Mol Basis Dis. 2017;1863:2307-18.

32. Liu CM, Ma JQ, Sun YZ. Puerarin protects rat kidney from lead-induced apoptosis by modulating the PI3K/Akt/eNOS pathway. Toxicol Appl Pharmacol. 2012;258:330-42.

33. Song XB, Liu G, Wang ZY, Wang L. Puerarin protects against cadmium-induced proximal tubular cell apoptosis by restoring mitochondrial function. Chem Biol Interact. 2016;260:219-31.

34. Kao ST, Wang SD, Lin CC, Lin LJ. Jin, Gui. Shen Qi Wan, a traditional Chinese medicine, alleviated allergic airway hypersensitivity and inflammatory cell infiltration in a chronic asthma mouse model. J Ethnopharmacol. 2018;227:181-90.

\section{Figures}

A
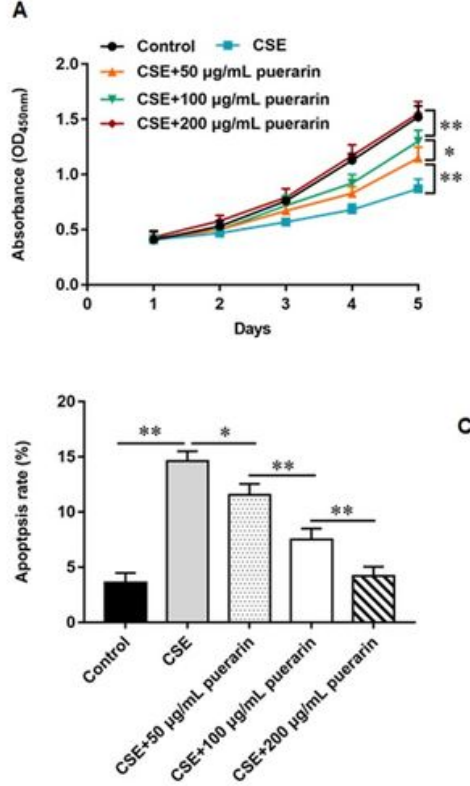

B
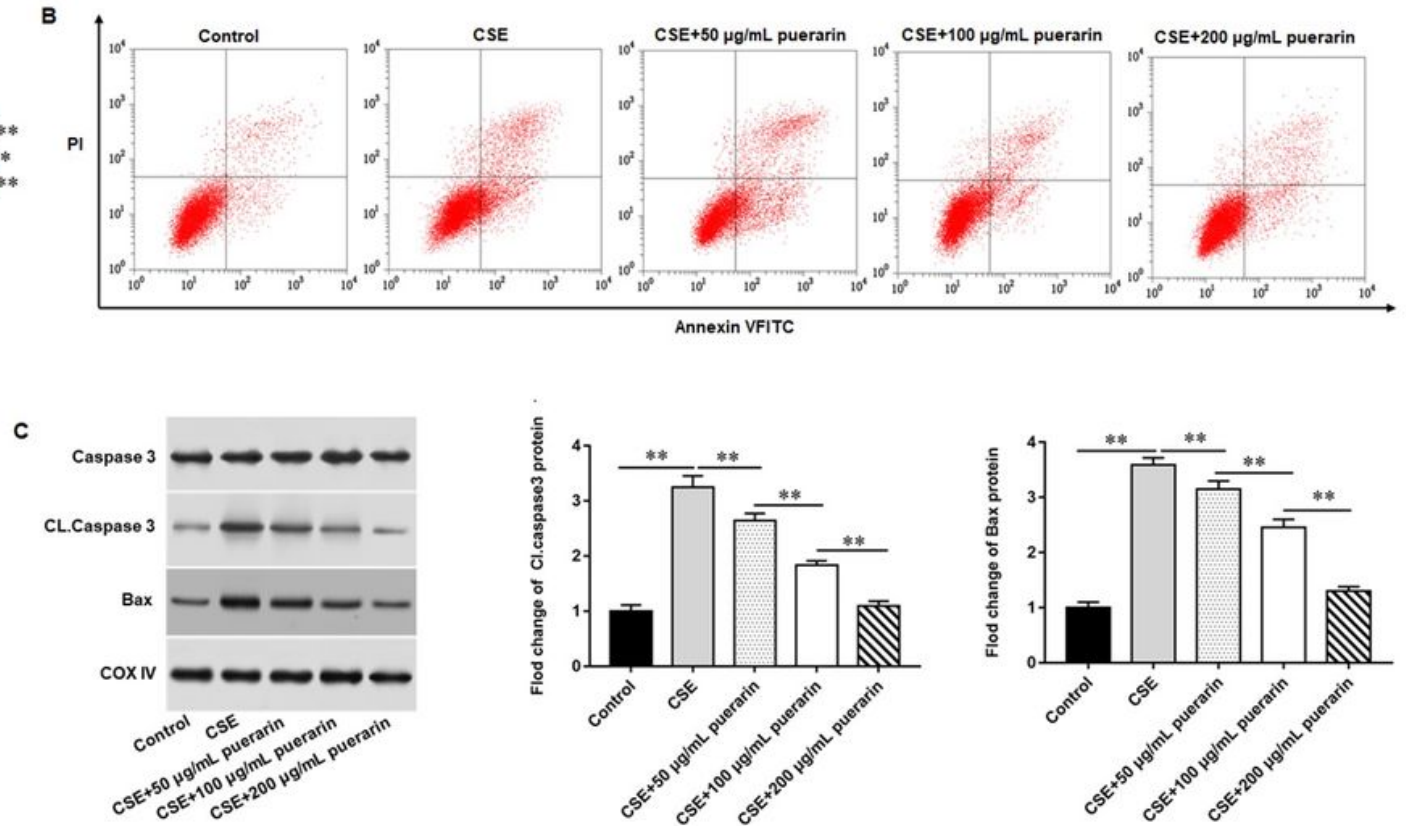
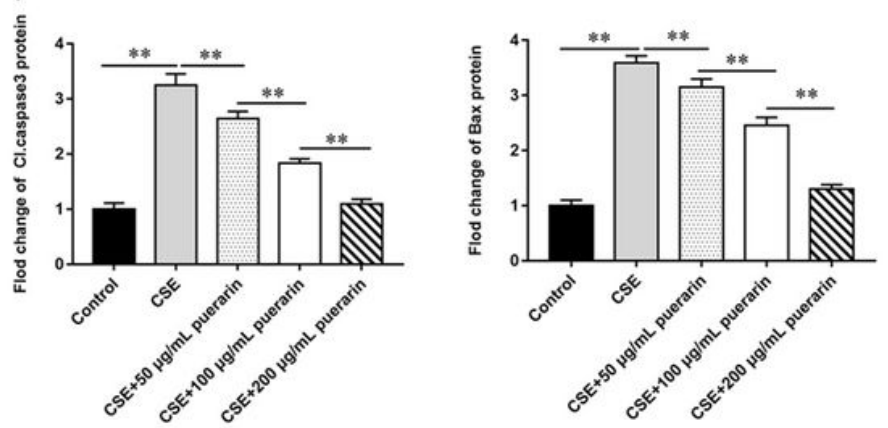

Figure 1 
Puerarin promotes the proliferation and inhibits apoptosis of CSE-induced HBECs. HBECs was induced by
$20 \%$ CSE and intervened with $50 \mu \mathrm{g} / \mathrm{mL}, 100 \mu \mathrm{g} / \mathrm{mL}$ and $200 \mu \mathrm{g} / \mathrm{mL}$ puerarin for $12 \mathrm{~h}$, respectively. (A)
MTT assay was used to measure the viability of CSE-induced HBECs. (B) Apoptosis of CSE-induced
HBECs were detected by flow cytometry. (C) The protein expression of Cleaved caspase3 and Bax in CSEPuerarin promotes the proliferation and inhibits apoptosis of CSE-induced HBECs. HBECs was induced by
$20 \%$ CSE and intervened with $50 \mu \mathrm{g} / \mathrm{mL}, 100 \mu \mathrm{g} / \mathrm{mL}$ and $200 \mu \mathrm{g} / \mathrm{mL}$ puerarin for $12 \mathrm{~h}$, respectively. (A)
MTT assay was used to measure the viability of CSE-induced HBECs. (B) Apoptosis of CSE-induced
HBECs were detected by flow cytometry. (C) The protein expression of Cleaved caspase3 and Bax in CSEPuerarin promotes the proliferation and inhibits apoptosis of CSE-induced HBECs. HBECs was induced by
$20 \%$ CSE and intervened with $50 \mu \mathrm{g} / \mathrm{mL}, 100 \mu \mathrm{g} / \mathrm{mL}$ and $200 \mu \mathrm{g} / \mathrm{mL}$ puerarin for $12 \mathrm{~h}$, respectively. (A)
MTT assay was used to measure the viability of CSE-induced HBECs. (B) Apoptosis of CSE-induced
HBECs were detected by flow cytometry. (C) The protein expression of Cleaved caspase3 and Bax in CSEPuerarin promotes the proliferation and inhibits apoptosis of CSE-induced HBECs. HBECs was induced by
$20 \%$ CSE and intervened with $50 \mu \mathrm{g} / \mathrm{mL}, 100 \mu \mathrm{g} / \mathrm{mL}$ and $200 \mu \mathrm{g} / \mathrm{mL}$ puerarin for $12 \mathrm{~h}$, respectively. (A)
MTT assay was used to measure the viability of CSE-induced HBECs. (B) Apoptosis of CSE-induced
HBECs were detected by flow cytometry. (C) The protein expression of Cleaved caspase3 and Bax in CSEinduced HBECs were analyzed by Western botting. $\beta$-actin was used as an invariant internal control for calculating protein-fold changes. $N=3,{ }^{\star} P<0.05,{ }^{\star *} P<0.01$.

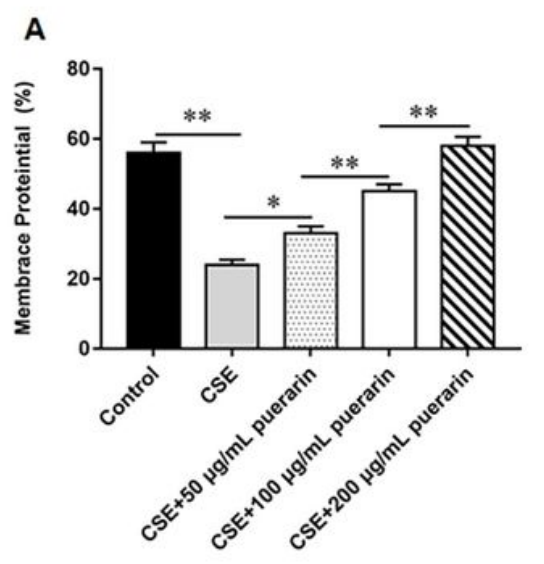

D

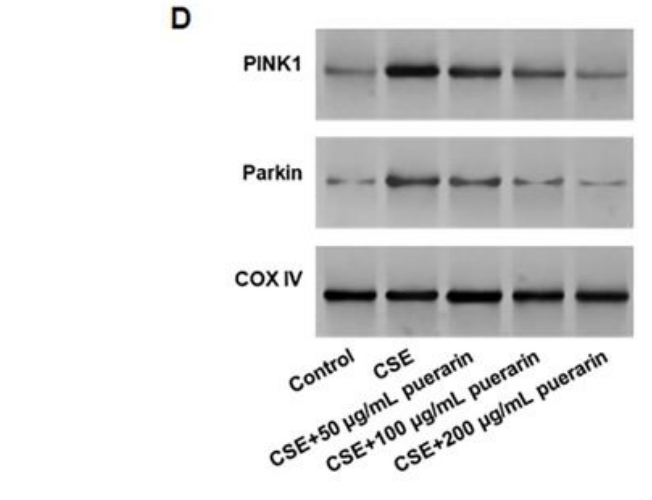

B

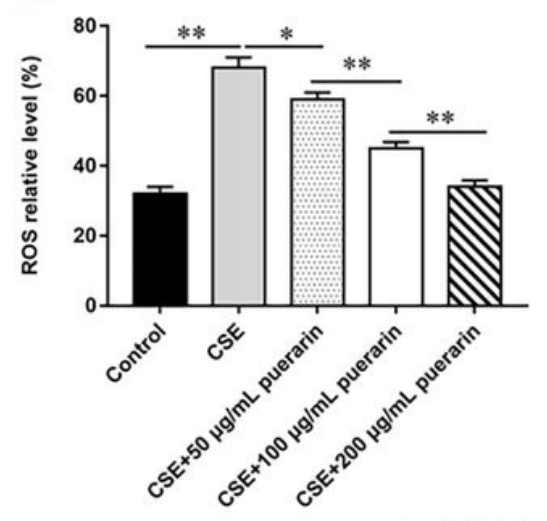

- Control $\square$ CSE CSE $+50 \mu \mathrm{g} / \mathrm{mL}$ puerarin $\square \mathrm{CSE}+100 \mu \mathrm{g} / \mathrm{mL}$ puerarin $\Delta \mathrm{CSE}+200 \mu \mathrm{g} / \mathrm{mL}$ puerarin

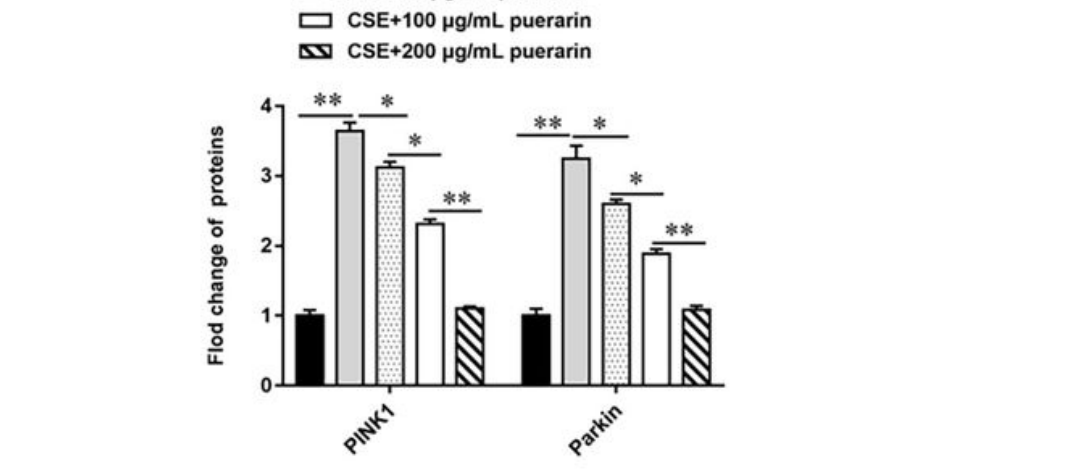

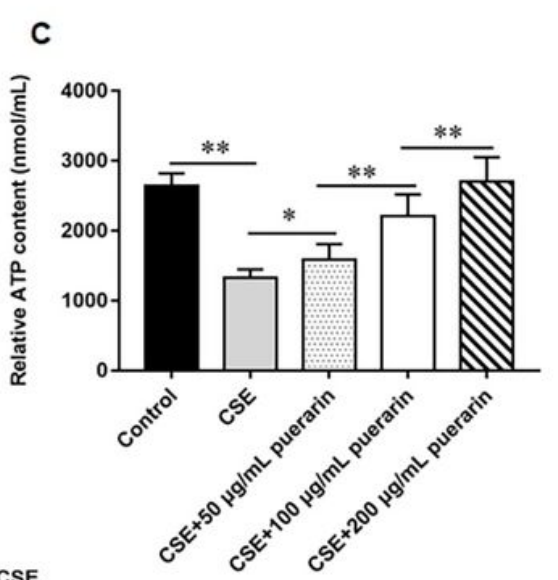

$c^{5} \quad c^{\sum^{x}} \quad c^{5^{2}}$ Puerarin promotes the proliferation and inhibits apoptosis of CSE-induced HBECs. HBECs was induced by
$20 \%$ CSE and intervened with $50 \mu \mathrm{g} / \mathrm{mL}, 100 \mu \mathrm{g} / \mathrm{mL}$ and $200 \mu \mathrm{g} / \mathrm{mL}$ puerarin for $12 \mathrm{~h}$, respectively. (A)
MTT assay was used to measure the viability of CSE-induced HBECs. (B) Apoptosis of CSE-induced
HBECs were detected by flow cytometry. (C) The protein expression of Cleaved caspase3 and Bax in CSE-

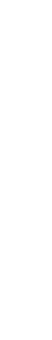

\section{Figure 2}

Puerarin inhibits mitochondrial autophagy of CSE-induced HBECs. HBECs was induced by $20 \%$ CSE and intervened with $50 \mu \mathrm{g} / \mathrm{mL}, 100 \mu \mathrm{g} / \mathrm{mL}$ and $200 \mu \mathrm{g} / \mathrm{mL}$ puerarin for $12 \mathrm{~h}$, respectively. (A) Flow cytometry (JC-1) was used to detect changes in MMP level in CSE-induced HBECs. (B) Mitochondrial ROS levels were detected by flow cytometry (DCFH-DA). (C) The content of ATP in CSE-induced HBECs was detected with kits. (D) Western botting was used to detect the expression of mitochondrial autophagy-related proteins such as PINK1 and Parkin. $\beta$-actin was used as the loading control. $N=3,{ }^{\star} P<0.05 \mathbb{Z}^{\star \star} P<0.01$. 

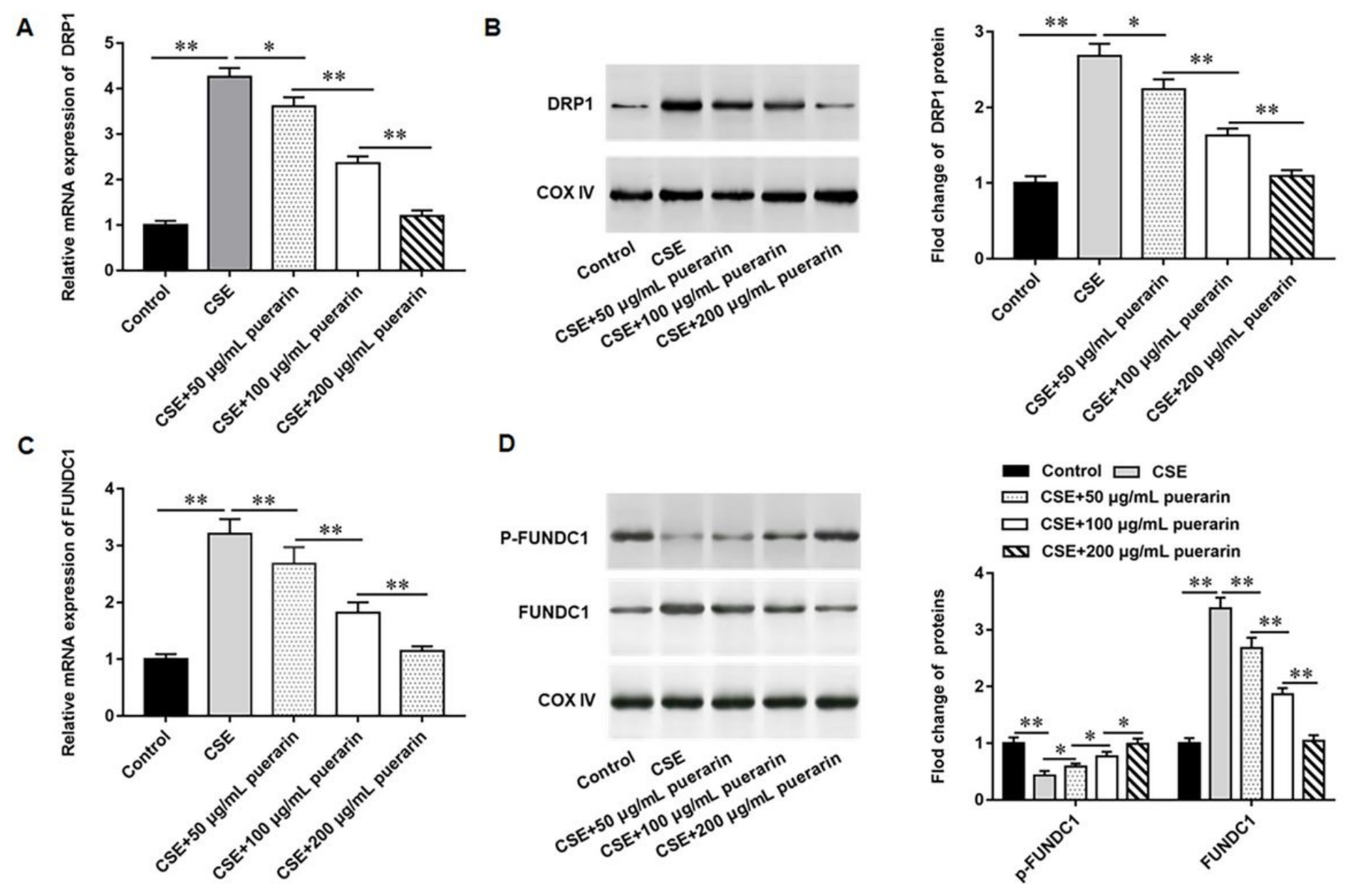

\section{Figure 3}

Puerarin down-regulates the expression of DRP1 and FUNDC1. HBECs was induced by $20 \%$ CSE and intervened with $50 \mu \mathrm{g} / \mathrm{mL}, 100 \mu \mathrm{g} / \mathrm{mL}$ and $200 \mu \mathrm{g} / \mathrm{mL}$ puerarin for $12 \mathrm{~h}$, respectively. (A) Relative mRNA expression of DRP1 was analyzed by RT-qPCR. (B) Western botting was used to measure the protein expression of DRP1. (C) Relative expression of FUNDC1 was detected by RT-qPCR. (D) The protein expression of FUNDC1 and p-FUNDC1 in CSE-induced HBECs were analyzed by Western botting. $\beta$-actin was used as an internal reference. $N=3, * P<0.05, * * P<0.01$. 
A

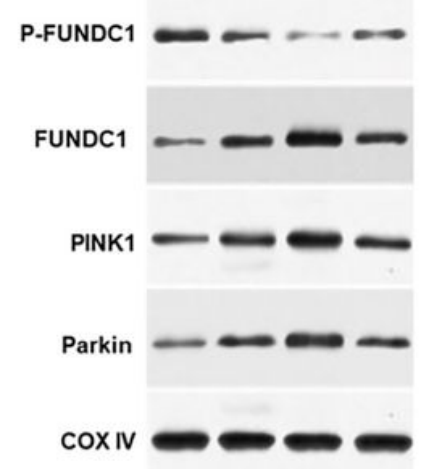

C

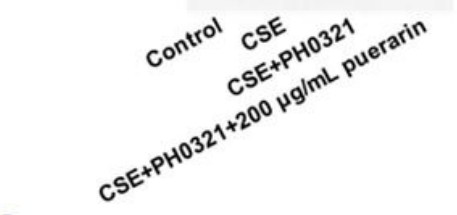

Control $\square$ CSE $\square$ CSE+PH0321 CSE $\mathrm{CSH} 0321+200 \mu \mathrm{gg} / \mathrm{mL}$ puerarin

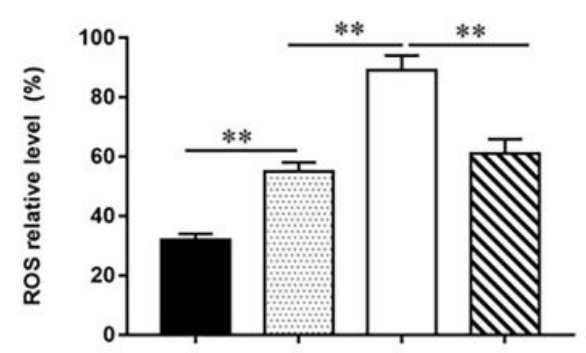

- Control $\square$ CSE $\square$ CSE+PH0321 $\triangle \mathrm{CSE}+\mathrm{PH} 0321+200 \mu \mathrm{g} / \mathrm{mL}$ puerarin

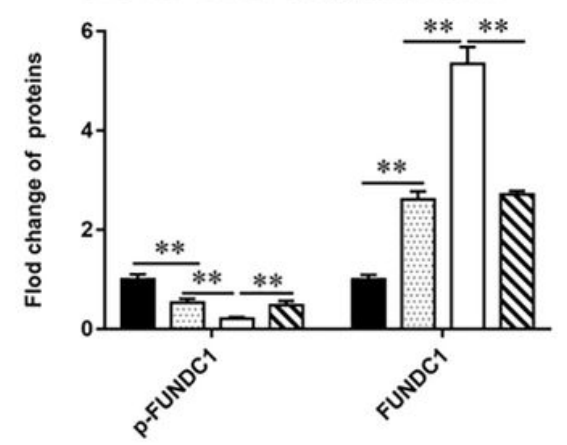

D

- Control $\square$ CSE 口 CSE+PH0321 W CSE+PH0321+200 $\mu \mathrm{g} / \mathrm{mL}$ puerarin

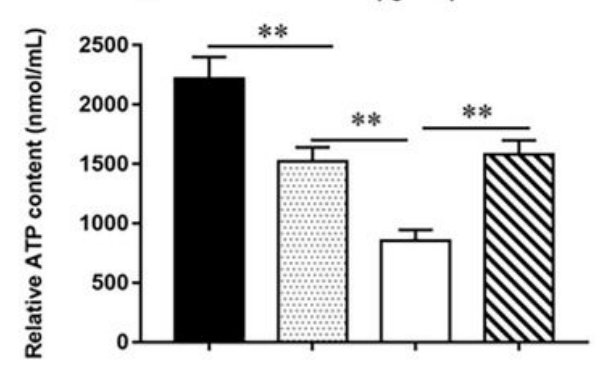

B

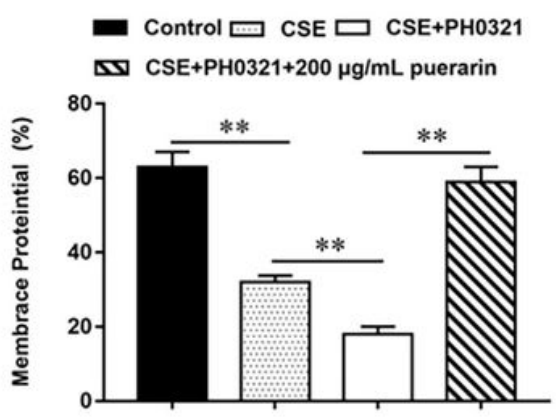

E - Control $\mathrm{D}$ CSE 口 CSE+PH0321 CSE+PH0321+200 $\mu \mathrm{g} / \mathrm{mL}$ puerarin

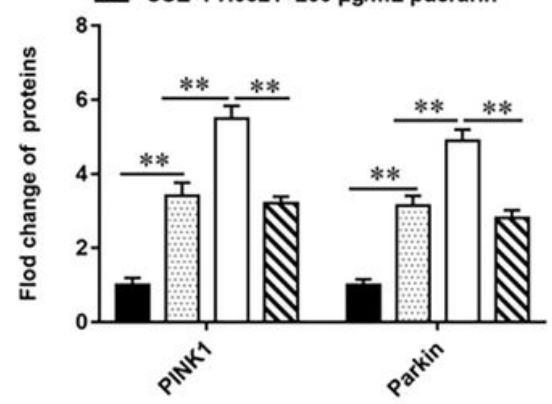

\section{Figure 4}

Puerarin prevents mitochondrial autophagy by inhibiting dephosphorylation of FUNDC1. PH0321, a protein phosphatase inhibitor, treated $20 \%$ CSE-induced HBECs and then co-cultured with puerarin at the concentration of $200 \mu \mathrm{g} / \mathrm{mL}$. (A) The protein expression of FUNDC1 and p-FUNDC1 in CSE-induced HBECs were analyzed by Western botting. (B) Flow cytometry (JC-1) was used to detect changes in mitochondrial membrane potential in CSE-induced HBECs. (C) Mitochondrial ROS levels were detected by flow cytometry (DCFH-DA). (D) The content of ATP in CSE-induced HBECs was detected with kits. (E) Western botting was used to detect the expression of mitochondrial autophagy-related proteins such as PINK1 and Parkin. $\beta$-actin was used as an invariant internal control for calculating protein-fold changes. $\mathrm{N}=3$, ** $\mathrm{P}<0.01$. 
A

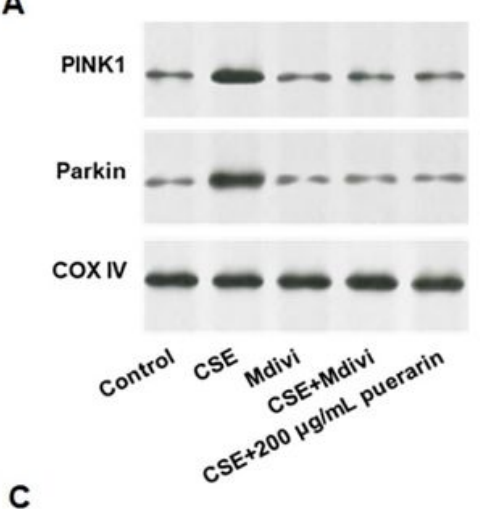

$\square$ Control $\square$ CSE $\square$ Mdivi $\square$ CSE+Mdivi $\Delta \mathrm{CSE}+200 \mu \mathrm{g} / \mathrm{mL}$ puerarin

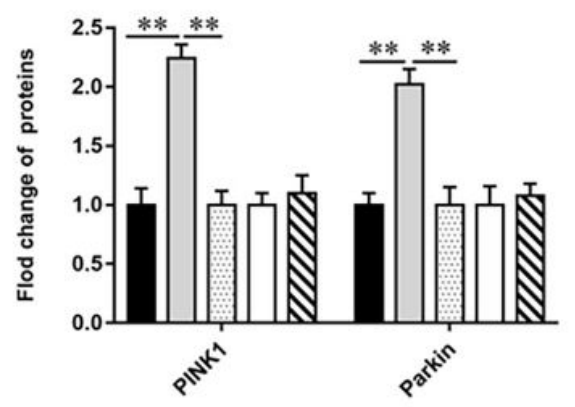

B

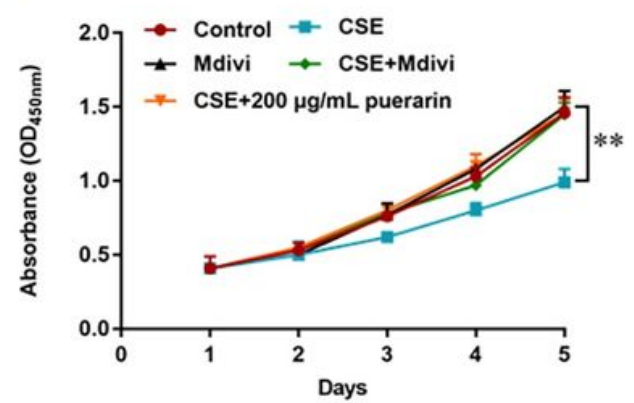

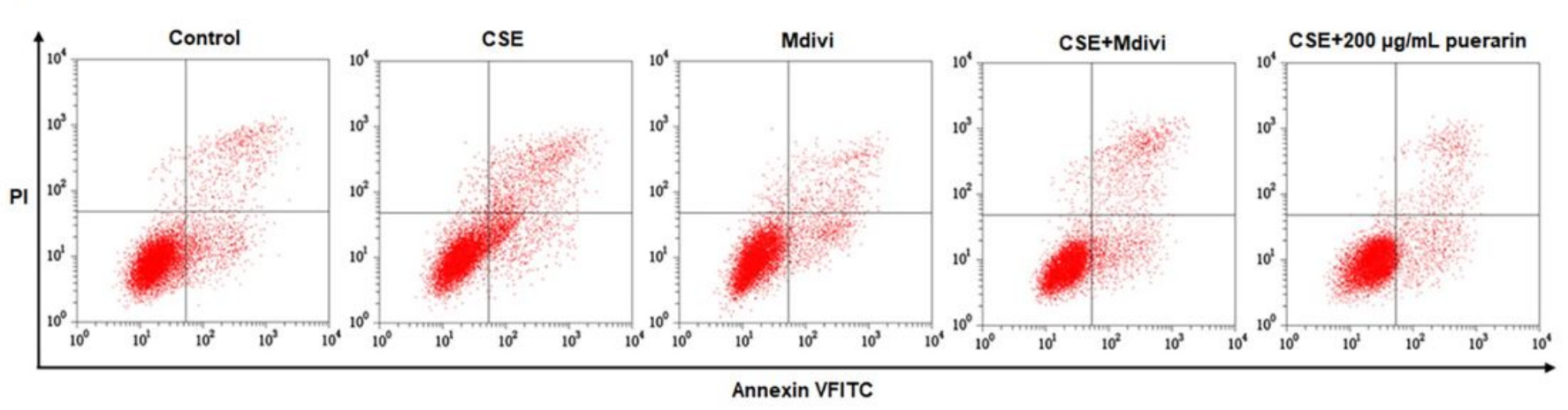

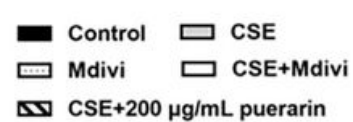

$\Delta \mathrm{CSE}+200 \mu \mathrm{g} / \mathrm{mL}$ puerarin

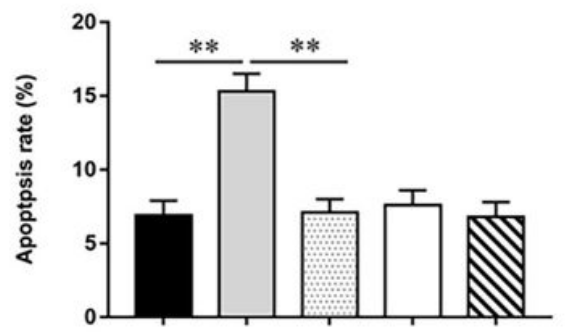

D

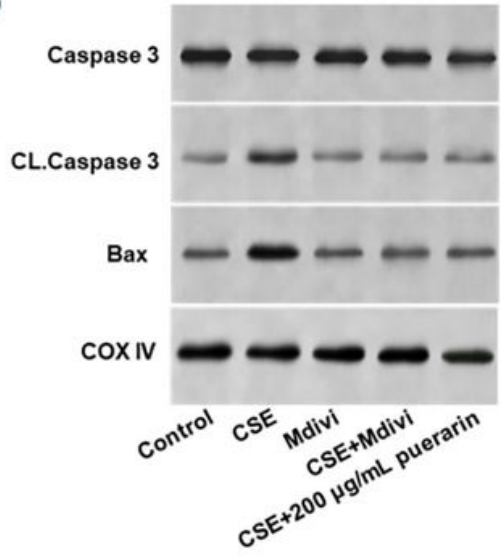

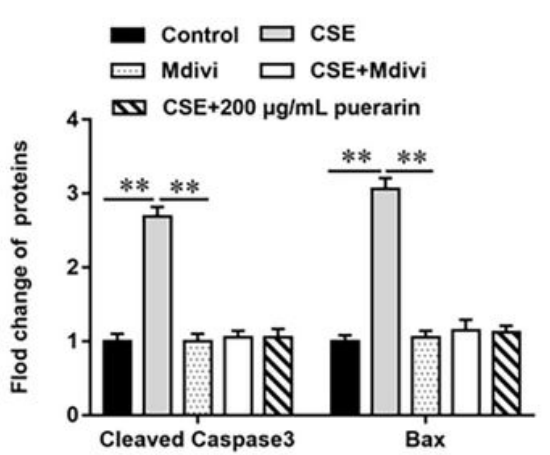

\section{Figure 5}

Puerarin inhibits apoptosis of CSE-induced HBECs by inhibiting mitochondrial autophagy. Mdivi, a mitochondrial autophagy inhibitor, treated 20\% CSE-induced HBECs and then co-cultured with puerarin at a concentration of $200 \mu \mathrm{g} / \mathrm{mL}$. (A) Western botting was used to detect the expression of mitochondrial autophagy-related proteins such as PINK1 and Parkin. (B) MTT assay was used to measure the viability of CSE-induced HBECs. (C) Apoptosis of CSE-induced HBECs were detected by flow cytometry. (D) The protein expression of Cleaved caspase 3 and Bax in CSE-induced HBECs were analyzed by Western botting. $\beta$-actin was used as the loading control. $N=3$, ${ }^{\star \star} P<0.01$. 
A

C

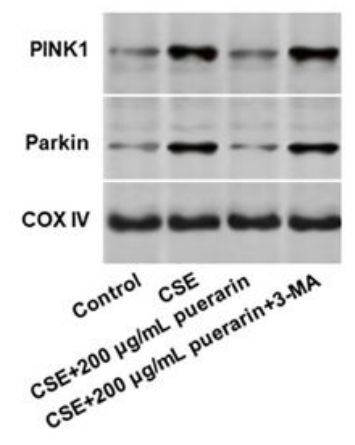

B

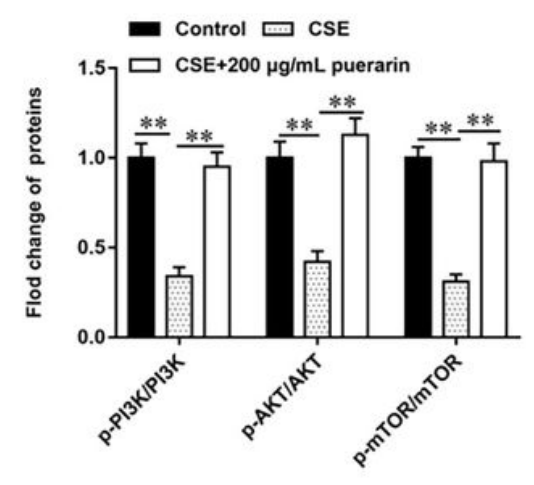

D

- Control $\square$ cSE 口 $\mathrm{CSE}+200 \mu \mathrm{g} / \mathrm{mL}$ puerarin $\Delta \mathrm{CSE}+200 \mu \mathrm{g} / \mathrm{mL}$ puerarin+3-MA

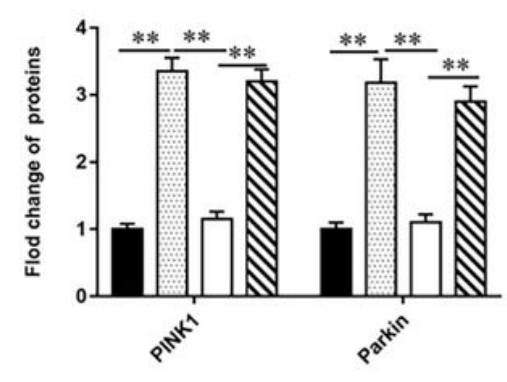

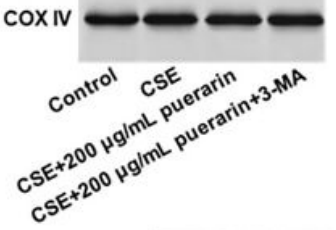

PI3K
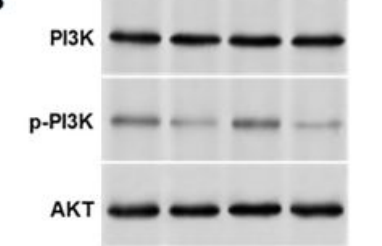

P-AKT $-\cdots$
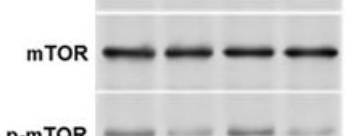

p-mTOR

$\cos$
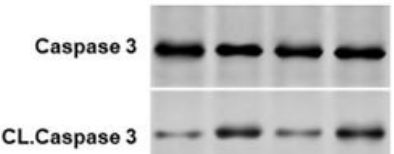

CL.Caspase $3 \ldots-\ldots$

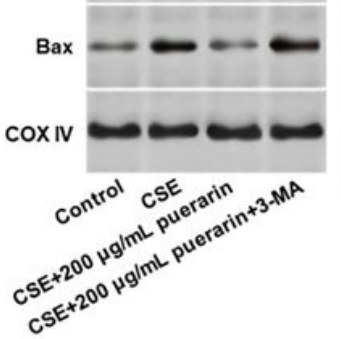

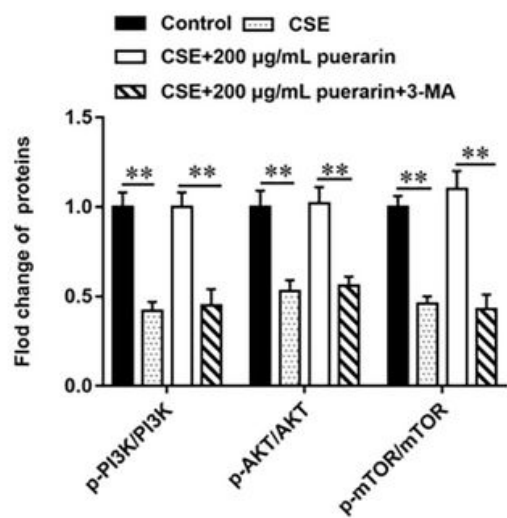

- Control $\mathrm{D}$ CSE

$\square \mathrm{CSE}+200 \mu \mathrm{g} / \mathrm{mL}$ puerarin

$\triangle \mathrm{CSE}+200 \mu \mathrm{g} / \mathrm{mL}$ puerarin+3-MA

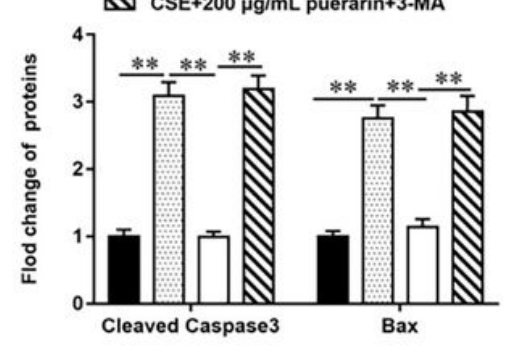

Figure 6

Puerarin inhibits mitochondrial autophagy and apoptosis in CSE-induced HBECs by activating the $\mathrm{PI}$ KK/AKT/mTOR signaling pathway. 3-MA, an inhibitor of PI3K, was added to $20 \%$ CES-induced HEBCs, which treated with puerarin at a concentration of $200 \mu \mathrm{g} / \mathrm{mL}$. (A-B) The protein expression of total PI3K, p-PI3K, total AKT, p-AKT, total mTOR and p-mTOR in CSE-induced HBECs were analyzed by Western botting. (C) Western botting was used to detect the expression of mitochondrial autophagy-related proteins such as PINK1 and Parkin. (D) The protein expression of Cleaved caspase3 and Bax in CSEinduced HBECs were analyzed by Western botting. $\beta$-actin was used as the loading control. $N=3$, ** $P<0.01$. 